\title{
«Stop the war on obesity!»
}

\section{Jean-Claude Vuille}

Prof. em. für Sozialpädiatrie, Facharzt Prävention und Gesundheitswesen, Mitglied FMH
Chronische Krankheiten, NCD (non-communicable diseases), verursachen heute $80 \%$ der Gesundheitskosten. Der unaufhaltsame Anstieg dieser Kosten veranlasste Bund und Kantone nun zur Lancierung einer nationalen Strategie zur Prävention der NCD. Das theoretische Grundlagenpapier liegt vor, konkrete Massnahmen sollen Ende Jahr vorgestellt und ab 2017 implementiert werden [1]. Auch wenn Massnahmen zur Verbesserung der Lebensbedingungen angestrebt werden, ist angesichts der politischen Machtverhältnisse zu befürchten, dass das Ganze am Schluss hauptsächlich auf eine «Stärkung der Selbstverantwortung für die Gesundheit» hinauslaufen wird. Selbstverantwortung für die Gesundheit ist gut und wichtig und wird von den meisten Schweizerinnen und Schweizern auch gelebt. Staatlich angemahnte Selbstverantwortung ist für Menschen in schwierigen Lebenssituationen aber kontraproduktiv und ethisch fragwürdig [2, 3]. Versuche, die Internet-basierte (Des-)Informationsflut durch öffentliche Kampagnen zu übertönen, werden ohnehin kaum nachhaltige Wirkungen entfalten können. Wenn eine solche Strategie doch erfolgreich sein sollte, würden die betroffenen Menschen länger leben und die Sozialversicherungen stärker belasten. Damit sollte der Versuch einer ökonomischen Legitimation vom Tisch sein. Im Strategiepapier wird wie schon bei früheren Kampagnen die Bedeutung von Ernährung und Bewegung besonders hervorgehoben. Implizit wird damit sicher auch die in der Schweiz wie in fast allen anderen Ländern zunehmende Prävalenz der Adipositas angepeilt [4]. Da zu den Ursachen der Adipo-

\section{Résumé}

L'obésité est précurseur de diverses maladies chroniques qui génèrent aujourd'hui $80 \%$ des dépenses totales en matière de santé en Suisse. Depuis les années 1980, la fréquence de la surcharge pondérale a drastiquement augmenté dans le monde. Jusqu'à présent, aucun pays n'a encore réussi à enrayer le phénomène qui s'explique communément par le fait que les personnes mangent de plus en plus et bougent de moins en moins. Le présent article propose une discussion et une réflexion critique de cette hypothèse sous l'angle mathématique, biologique, social et éthique. Les appels officiels à la responsabilité individuelle en matière de santé ont un effet discriminant et contre-productif. Au lieu d'une prévention comportementale, l'auteur plaide pour une prévention contextuelle à tous les niveaux. sitas im Einzelfall und der aktuellen Pandemie in der Öffentlichkeit und leider auch in der Fachwelt häufig sehr rudimentäre und die betroffenen Menschen diskriminierende Vorstellungen vorherrschen, wäre es wünschenswert, dass zu diesem Thema eine Debatte auf wissenschaftlicher Basis geführt würde [5]. Präventive Massnahmen müssen genau so wie kurative Leistungen evidence-based sein, sonst stiften sie mehr Schaden als Nutzen. In der Folge werden hier die wichtigsten wissenschaftlich belegten biologischen Abläufe und externen Risikofaktoren in geraffter Form in Erinnerung gerufen. Eine vollständige Literaturübersicht ist angesichts der unüberschaubaren Fülle einschlägiger Publikationen nicht möglich. Ergänzungen und Kritik sind deshalb willkommen!

\section{An der Regulation des Körpergewichts ist der ganze Organismus beteiligt}

Im Gehirn befindet sich die Steuerungszentrale mit vielen Input- und Output-Signalen. Eine ganze Reihe von Hormonen spielt mit: Thyroxin, das Hungerhormon Ghrelin, das Sättigungshormon Leptin, Adiponectin, Insulin, Somatostatin, Glukagon, Cortisol u.a. Bei Adipösen wurden insbesondere die folgenden Unterschiede zu Normalgewichtigen nachgewiesen:

- Mikrobiom: Die bakterielle Zusammensetzung der Darmflora ist bei Adipösen weniger vielfältig als bei Normalgewichtigen. Auf welchem Weg dies zu veränderten Stoffwechselvorgängen und grösserer Tendenz zu Fettablagerung führt, ist noch unklar. Sicher spielt das Immunsystem eine wichtige Rolle [6].

- Leptin-Resistenz: Das Hormon Leptin wird hauptsächlich von Adipozyten exprimiert. Es unterdrückt im Gehirn das Hungergefühl. Viele Adipöse empfinden aber trotz hohem Leptinspiegel kein echtes Sättigungsgefühl, es liegt eine Leptinresistenz vor.

- Thermogenese vs. Fettspeicherung: Bei den meisten Erwachsenen, auch bei Adipösen, bleibt das Körpergewicht über längere Zeiträume ziemlich konstant. Diese Konstanz kann mit dem einfachen Energiebilanzmodell (Nahrungskalorien minus Grundumsatz minus Muskelaktivität) nicht erklärt werden (siehe disclosure). Die Feinsteuerung geschieht durch die Thermogenese in den braunen Fettzellen 


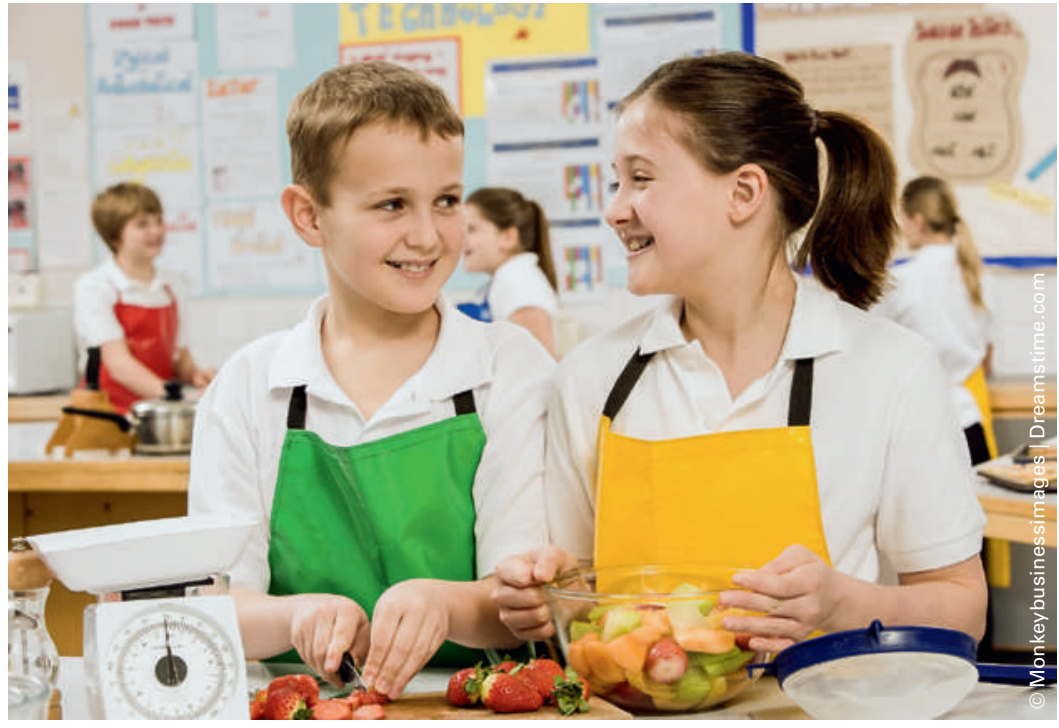

Bereits Kinder sollen lernen, sich mit ausgewogener Ernährung auseinanderzusetzen. Gesunde Zwischenmahlzeiten aus qualitativ hochwertigen Nahrungsmitteln tragen zu einem optimalen Körpergewicht bei.

und in den Muskeln: Verbrennung von Substrat in sogenannten "futile cycles", Abgabe der Energie in Form von Wärme. Man muss davon ausgehen, dass zur Aufrechterhaltung der Homöostase unter dem Einfluss des Lebensstils und externer Faktoren ständig und automatisch eine feine Abstimmung zwischen Fett-Einlagerung, Fett-Mobilisierung und Verbrennung stattfindet. In jedem Lebensalter kann das Ziel dieser Gewichts-Homöostase durch besondere Lebensumstände angehoben werden; es kommt zu einer oft therapieresistenten Adipositas.

\section{Für die Prävention ist die Qualität der Nahrung wichtiger als die Quantität.}

\section{Genetische Veranlagung}

In den 1960er Jahren belegten die ersten Zwillings- und Adoptivkinderstudien die grosse Bedeutung der erblichen Anlagen bei der Entstehung der Adipositas. Dank der modernen Gentechnik konnten verschiedene einschlägige Gene identifiziert werden, und es ist anzunehmen, dass die meisten der beschriebenen biologischen Vorgänge durch spezifische Gene determiniert werden [7]. Das traditionelle Verständnis der Erbsubstanz konnte das Phänomen der AdipositasPandemie mit Beginn in den 1980er Jahren allerdings nicht erklären, da sich die DNA einer Bevölkerung nicht innerhalb einer Generation grundlegend verändert. Die Entdeckung epigenetischer Mechanismen lässt nun aber die Vermutung zu, dass veränderte Lebensumstände vermehrt einzelne Gene aktivieren, resp. blockieren [8].

\section{Risikofaktoren}

\section{Perinatale Einflüsse}

Übergewicht, Folsäuremangel, aber auch der Konsum künstlich gesüsster Getränke der Mutter während der Schwangerschaft erhöhen das Risiko, dass das Kind später übergewichtig wird. Mit Kaiserschnitt geborene Kinder sind später doppelt so häufig übergewichtig als vaginal geborene, wahrscheinlich weil sich nur bei einer vaginalen Geburt ein normales Mikrobiom im Darm des Kindes entwickeln kann [9].

\section{Lebensstil}

Ernährung: Ungehemmter Kalorienkonsum kann die Kapazität des Körpers zur Verbrennung überflüssiger Kalorien überfordern. Warum essen Menschen zu viel? Eine mögliche biologische Erklärung liefert die oben beschriebene Leptin-Resistenz, deren Ursache aber unklar bleibt. Für die Prävention ist die Qualität der Nahrung wichtiger als die Quantität. Der Zucker in Süssgetränken wird von vielen Autoren als eine der wichtigsten Ursachen der Adipositas angesehen. Als in den USA in den frühen 1980er Jahren die Saccharose im Coca Cola durch die billigere aus Maissirup gewonnene Fruktose ersetzt wurde, begann gleichzeitig die Prävalenz der Adipositas massiv zuzunehmen [10] Fruktose mündet im Gegensatz zur Glukose tatsächlich direkt in einen adipogenen Stoffwechselzyklus [11] Laut neuesten Studien können auch künstliche Süssstoffe zu übermässiger Gewichtszunahme führen.

Bewegung: Muskelaktivität hilft grundsätzlich zur Erhaltung eines gesunden Körpergewichts. Auffallend ist, dass in den meisten Studien nur die körperliche Aktivität in der Freizeit und im Alltag (Arbeitsweg, Treppe statt Lift, Sport etc.) berücksichtigt wird. Bauarbeiter und Müllentsorger sind trotz sehr hohem Energieverbrauch nicht schlanker als die Durchschnittsbevölkerung. Schlaf und künstliches Licht: Sowohl zu wenig ( $<6 \mathrm{~h})$ als auch zu viel Schlaf ( $>9$ h) geht mit einem erhöhten Risiko für Übergewicht einher. Exposition für künstliches Licht in der Nacht ist wahrscheinlich ein unabhängiger Risikofaktor [13]. Bildschirm-bezogene Aktivitäten (TV, Handy, Internet): Ein statistischer Zusammenhang wurde in vielen Studien gefunden, aber wegen ungenügend kontrollierter Störfaktoren wird bezweifelt, dass hier eine einfache Ursache-Wirkungs-Beziehung vorliegt.

(Zu) hohe Raumtemperatur: In verschiedenen experi mentellen Studien wurde eine erhöhte Thermogenese im braunen Fettgewebe bei kühler Umgebungstemperatur nachgewiesen. Daraus wird spekuliert, dass die hohen Temperaturen in modernen Wohnungen die Entwicklung einer Adipositas begünstigen könnten. 


\section{Psychologie}

Stress: Dass chronischer Stress bei entsprechend disponierten Individuen Übergewicht bewirken kann, ist seit langem bekannt. Der bekannte englische Epidemiologe Michael Marmot wies nach, dass ein stressinduzierter Schmerbauch auch ohne erhöhten Kalorienkonsum entstehen kann. Die Erklärung liefert das Stresshormon Cortisol, das direkt eine abdominale Fettablagerung fördert.

\section{Vor hundert Jahren waren die Kinder der Oberschicht schwerer als die Kinder der Unterschicht.}

Psychiatrische Krankheiten: Anorexie und Hyperphagie kommen als Begleitsymptome bei verschiedenen psychischen Leiden vor. Adipositas tritt häufig als Nebenwirkung gewisser Psychopharmaka auf.

Psychosoziale Belastungen bei Kindern: Die Psychoanalytikerin Hilde Bruch beschrieb schon in den 1940er Jahren einen Zusammenhang zwischen dem Übergewicht bei Kindern und einer gestörten Eltern-KindBeziehung. Resultate von zwei longitudinalen Kohortenstudien bei Schulkindern zeigten übereinstimmend, dass Kinder, deren BMI im Verlauf der Schulzeit stark zunahm, viel häufiger mit bedeutenden psychosozialen Problemen zu kämpfen hatten als Normalgewichtige oder konstant Übergewichtige.

\section{Soziales}

Sozialschicht: In den meisten entwickelten Ländern besteht heute ein markanter sozialer Gradient der Adipositas-Prävalenz zu Ungunsten der bildungsfernen Bevölkerungsgruppen. Das war nicht immer so: Vor hundert Jahren waren die Kinder der Oberschicht schwerer als die Kinder der Unterschicht. In Schwellenund Entwicklungsländern galt dies noch bis vor kurzem ebenfalls - Körperfülle als Privileg der Bessergestellten, als Symbol von Reichtum und Macht. In Chinas Grossstädten hat sich erst kürzlich ein drastischer Umbruch ereignet: In der neureichen jeunesse dorée beginnt sich dort ein extremer Schlankheitswahn auszubreiten. Und ein anekdotischer Einschub aus der Schweiz:

«Das Einzige, womit Erika glänzen könnte, ist ihre Tochter. Aber diese Tochter ist ein lebender Vorwurf, weil sie dick ist, was in dieser Gesellschaft das Schlimmste überhaupt ist, schlimmer als drogensüchtig, das hat mir einmal eine Mutter vom Zürichberg gesagt.»

(Milena Moser im Interview zu ihrem Buch Das wahre Leben, Der Bund, 3.9.2013)

Klima in der Schule und am Arbeitsplatz: Laut einer Studie in der Stadt Bern kann ein gutes Schulklima die
Häufigkeit von Essstörungen reduzieren. Analoges dürfte für das Arbeitsplatzklima der Erwachsenen gelten.

\section{Physikalische und chemische Umwelt}

"Walkability»: In den USA konnte der Anstieg der Adipositas-Prävalenz in fussgängerfreundlichen Wohngegenden im Gegensatz zu verkehrsreichen Gegenden gestoppt werden.

Luftverschmutzung: Kürzlich wurde in der Schweiz bei einer Aufschlüsselung nach Wohnquartieren ein $\mathrm{Zu}$ sammenhang der Häufigkeit von Adipositas mit dem Grad der Luftverschmutzung nachgewiesen.

Lärm: Bewohner von lärmigen Quartieren haben ein höheres Adipositasrisiko [13].

Diverse Schadstoffe: Vor allem die Exposition für Bisphenol A - eine Chemikalie, die weltweit in grossen Mengen hergestellt und in der Produktion von PlastikGegenständen und von Epoxidharzen verwendet wird - steht im Verdacht, die Entstehung der Adipositas zu begünstigen [14]. Es wurden auch Zusammenhänge mit DDT-Abbauprodukten gefunden.

\section{Wo liegt die Ursache der weltweiten Adipositas-Epidemie?}

Warum ist in der Kurve der Adipositasprävalenz in den USA in den frühen 80er Jahren plötzlich ein deutlicher Knick nach oben aufgetreten, und warum wurde in der Folge in immer mehr Ländern eine ähnliche Tendenz festgestellt? Bisher hat niemand den Versuch unternommen, alle bis heute identifizierten Risikofaktoren gleichzeitig in einer multivariaten Studie zu berücksichtigen. Dazu wären riesige Stichproben und Mengen von historischen Daten nötig, die praktisch nicht zu beschaffen sind. Das bedeutet - leider -, dass gesichertes Wissen wohl nie vorliegen wird und wir uns damit begnügen müssen, unser Handeln auf möglichst plausible Hypothesen abzustützen. Der in diesem Artikel angehäufte Faktenberg könnte die Hypothese suggerieren, dass die Summe aller Risikofaktoren kontinuierlich angestiegen ist bis zu einem Schwellenwert, $a b$ dem die metabolischen Kompensationsmöglichkeiten bei immer mehr Menschen überschritten wurden. Die psychosoziale Hypothese weist auf die zunehmende Verunsicherung der Menschen hin, auf die Angst vor dem Verlust des Arbeitsplatzes und der Kontrolle über das eigene Leben, vor den Auswirkungen der Klimaerwärmung, des Terrorismus, der Völkerwanderung und der unkontrollierbaren Macht der Finanzindustrie und der Multis, die Wut auf angebliche Sündenböcke. Die Idee, dass ein Organismus in einer bedrohlichen Situation auf «Vorratshaltung» umstellt, ist biologisch 
nicht völlig abwegig. Dafür gibt es in der Natur genügend Beispiele. Andere Hypothesen sind denkbar.

\section{Schlussfolgerungen für die Praxis}

Das erste Gebot der Medizin im Falle mangelnder Evidenz heisst «primum nil nocere». Das bedeutet für das öffentliche Gesundheitswesen Verzicht auf jegliche Massnahmen, welche die Betroffenen stigmatisieren. Dazu gehören alle Kampagnen, welche sich direkt an die einzelnen Menschen richten und sie zu einer gesünderen Lebenshaltung nötigen. Diese Art von Verhaltensprävention ist erwiesenermassen nicht nur unwirksam [15], sondern direkt kontraproduktiv. Bei Durchsicht der aufgelisteten Risikofaktoren ergeben sich aber viele Ansatzpunkte für die Verhältnisprävention.

Schulen leisten bereits heute ihren Beitrag, z.B. durch den Abschied von Automaten mit Süssgetränken, durch die Weigerung, die Zahl der Turnlektionen zugunsten von mehr IT-Unterricht zu reduzieren, durch die Organisation von lustvollen Schulhaus-Znünis unter Mitwirkung der Eltern, durch ihren Einsatz für sichere fussgänger- und velofreundliche Schulwege, für ein gutes Schulklima etc. Es gibt Anhaltspunkte, dass dank dieses Engagements in der Schweiz die Zunahme der Adipositas-Prävalenz im Schulalter tatsächlich gebremst werden konnte.

Die Politik wäre gefordert, die soziale Ungleichheit zu reduzieren, statt sie mit dubiosen Steuerreformen weiter zu verstärken. Es wäre wichtig, die Luftverschmutzung und den Lärm in belasteten Wohngegenden zu bekämpfen, die Belastung mit Schadstoffen wie Bisphenol A zu überwachen, die Möglichkeit einer Konsumsteuer auf Süssgetränke zu prüfen, Eltern bei der Betreuung ihrer Kinder zu unterstützen, Frühförderprogramme für benachteiligte Familien zu finanzieren, Mittel für den freiwilligen Schulsport zur Verfügung zu stellen, die Zusammenarbeit der verschiedenen sozialen und medizinischen Organisationen und Institutionen auf Gemeinde- und Quartierebene zu fördern, etc. etc. Einige Punkte dieser Liste sind zwar Luftschlösser, könnten aber wenigstens zum Nachdenken anregen.

In der ärztlichen Praxis wirken Stirnrunzeln, Ermahnungen und Diätvorschriften eher kontraproduktiv. Konkrete Vorschläge zur Änderung der Lebensgewohnheiten haben nur Aussicht auf Erfolg, wenn der Patient / die Patientin von sich aus danach fragt. Als Erfolgskriterium sollte die Gewichtsabnahme eh nur bei Vorliegen einer sekundären Krankheit (Diabetes etc.) im Vordergrund stehen. Viel wichtiger ist die Lebensqualität als Übergewichtige/-r. Gynäkologen sollten die Folsäureversorgung der Schwangeren be- achten und von der Sectio caesarea ohne medizinische Indikation abraten. Das sind nur ein paar von vielen möglichen Präventionsmassnahmen im individuellen ärztlichen Kontakt.

\section{Verdankung}

Der Autor dankt der Leserschaft, die bis hier durchgehalten hat und über die Behauptungen und Fragen in diesem Artikel nachdenkt und debattiert.

\section{Disclosure statement}

Der Autor hat sich während seiner beruflich aktiven Zeit therapeutisch, präventiv und epidemiologisch, später als weiterhin Lernender mit der Adipositas bei Kindern beschäftigt. Er hat keine Mandate, weder vom Schweizerischen Bauernverband, noch von der Nahrungsoder Getränkeindustrie. Er hat auch kein persönliches Interesse an einer Verharmlosung der Adipositas. Sein eigener BMI lag während 65 Jahren als Erwachsener stets zwischen 20 und 24, was eine Anpassung von Kalorienaufnahme und -verbrauch mit einer Präzision von unter 1 Promille erforderte. Dies war nur mit äusserster Disziplin und täglicher minutiöser Kontrolle von Essen und Bewegung möglich (maximal tolerabler Fehler: 1,5 kcal/Tag). Wer's glaubt, ist selber schuld.

\section{Literatur}

1 Bruhin, E. Vorbeugen statt heilen: Nationale Strategie gegen nichtübertragbare Krankheiten. Spectra Mai 2016, S. 12.

2 Lewis S, Thomas SL, Hyde J, Castle D, Blood RW, Komesaroff PA. "I don't eat a hamburger and large chips every day!» A qualitative study of the impact of public health messages about obesity on obese adults. BMC Public Health. 2010 June 4;10:309. http:// www.biomedcentral.com/1471-2458/10/309.

3 Frank, A. Why is it so difficult to lose weight? Am J Lifestyle Med. 2014;8(5):318-23

4 Ng M, FlemingT, Robinson M, Thomson B, Graetz N, Margono C, et al. Global, regional, and national prevalence of overweight and obesity in children and adults during 1980-2013: a systematic analysis for the Global Burden of Disease Study 2013. The Lancet. 2014;384(9945):766-81.

5 Stenvinkel, P. Obesity - a disease with many aetiologies disguised in the same oversized phenotype. Has the overeating theory failed? Nephrol Dial Transplant.2015;30(10):1656-64.

6 Hartstra AV, Bouter KEC, Bäckhed F, Nieuwdorp M. Insights Into the Role of the Microbiome in Obesity and Type 2 Diabetes. Diabetes Care 2015;38(1):159-65. http://dx.doi.org/10.2337/dc14-0769.

7 Wardle J, Carnell S, Haworth, CMA, Robert Plomin, R. Evidence for a strong genetic influence on childhood adiposity despite the force of the obesogenic environment. Am J Clin Nutr. 2008;87(2):398-404.

8 Walter S, Mejía-Guevara I, Estrada K, Liu, SY, Glymour, MM. Association of a Genetic Risk Score With Body Mass Index Across Different Birth Cohorts. JAMA. 2016;316:63-9.

9 Huh SY, Rifas-Shiman SL, Zera CA, et al. Delivery by caesarean section and risk of obesity in preschool age children: a prospective cohort study. Arch Dis Child 2012;97(7):610-6.

10 Berneis K, Keller U. Steigender Fruktosekonsum als Auslöser des metabolischen Syndroms? Schweiz Med Forum. 2001;01(20): 523-36.

11 Perez-Pozo SE, Schold, J, Lopez Lillo J. Excessive fructose intake induces the features of metabolic syndrome in healthy adult men: role of uric acid in the hypertensive response. Int J Obesity. 2009; 34:454-61.

12 Rybnikova NA, Haim A, Portnov BA. Does artificial light-at-night exposure contribute to the worldwide obesity pandemic? Int J Obesity 2016 January 22; doi:10.1038/ijo.2015.255.

13 Nicole, W. Noise and Body Fat: Uncovering New Connections. Environ Health Perspect. 2016;124(3):329.

14 Bhandari R, Xiao J, Shankar A. Urinary Bisphenol A and obesity in US children. Am J Epidemiol. 2013;177(11):1263-70.

15 Fildes A, Charlton J, Rudisill C, Littlejohns P, Prevost AT, Gulliford, MC. Probability of an Obese Person Attaining Normal Body Weight: Cohort Study Using Electronic Health Records. Am J Public Health. 2015;105(9):e54-e59. 